\title{
HISTIOCYTIC MEDULLARY RETICULOSIS IN A CHILD
}

\author{
BY \\ MALCOLM FOWLER \\ From Adelaide Children's Hospital, Adelaide, South Australia
}

(RECEIVED FOR PUBLICATION MARCH 2, 1960)

Scott and Robb-Smith (1939), using the name 'histiocytic medullary reticulosis', reported four cases of a subacute illness characterized by fever, anaemia, leucopenia, jaundice and enlargement of the lymph nodes, spleen and liver. Microscopically there was a proliferation of phagocytes and giant cells in the reticulo-endothelial system, the affected tissues showing a tendency to undergo haemorrhage and necrosis. Marshall $(1956 \mathrm{a}, \mathrm{b})$ described eight cases and referred to seven others reported in the literature since 1939.

The disease has not been reported in children and it is for this reason that the following case is mainly of interest. (For a review of the literature and discussion of various aspects of the disease Marshall's publications, 1956a, 1956b, should be consulted.)

\section{Case Report}

The patient was admitted to the Adelaide Children's Hospital on October 9, 1954, at the age of 4 years 5 months with generalized enlargement of the lymph nodes.

Her previous health had been good, apart from chickenpox and tonsillectomy, until three months before admission when a small lump in the right side of the neck had been noticed by her parents. A month before admission, because of a sudden increase in size of her neck, she was taken to a general practitioner who found rubbery enlargement of five or six lymph nodes in the right side of the neck. He could not feel the spleen at this time. Following treatment with antibiotics the nodes subsided considerably. Two days before admission they enlarged again, accompanied by a fever with a temperature of $102^{\circ} \mathrm{F}$., tiredness and loss of appetite.

On admission to hospital the cervical, axillary and inguinal glands were all enlarged and the spleen was felt 3 fingers' breadth below the costal margin. The liver could not be felt. Blood examination showed $\mathrm{Hb}$ $10 \mathrm{~g} . / 100 \mathrm{ml}$., the red blood cells numbered $3,800,000$ per c.mm. and the white cells 8,800 per c.mm., $61 \%$ being neutrophils, $37 \%$ lymphocytes and $2 \%$ large lymphocytes. No abnormality was seen in a radiograph of the chest. A week after admission the Paul-Bunnell test gave agglutination at a serum dilution of 1 in 32, rising to 1 in 64 three days later. Another blood examination on the day she left hospital, 11 days after her admission, showed $\mathrm{Hb} 12 \mathrm{~g} . / 100 \mathrm{ml}$., a white cell count of 14,800 per c.mm. of which $45 \%$ were neutrophils, $35 \%$ lymphocytes, $18 \%$ monocytes and $2 \%$ abnormal lymphocytes. Apart from two short bursts of fever, up to $100^{\circ} \mathrm{F}$., her temperature was normal during her stay. On discharge from hospital her spleen could just be felt at the costal margin and the lymph nodes were much smaller. She was sent home to rest in bed, diagnosed as suffering from infectious mononucleosis.

On November 11, 1954, she returned to hospital with enlargement of the cervical lymph nodes which had apparently occurred over a few hours. Some fever had been noted five days before.

On examination she was pale and had very large fleshy lymph nodes in the right cervical region extending from behind the ear to the supraclavicular fossa. There was also enlargement of the nodes in the right axilla. The left cervical nodes were only slightly enlarged and no enlargement was felt in the inguinal regions. The spleen was enlarged to the level of the umbilicus but the liver could not be felt. The throat and middle ears were not inflamed.

Blood examination soon after admission showed $\mathrm{Hb} 10 \mathrm{~g} . / 100 \mathrm{ml}$. and a white cell count of 8,200 per c.mm.; bone marrow smears were normal. Further blood examinations during her illness showed a progressive fall in red and white cells and a later fall in platelets; Fig. 1 shows some of these changes. Reticulocytes never made up more than $2 \%$ except on one occasion when they reached $4 \%$ of the total red cell count. Four days after admission the lymph nodes were smaller but one from the right side of the neck was removed. Microscopical examination revealed that the general architecture of the node had been destroyed apart from some remaining cortical lymphoid follicles. In one part of the cortex was a wedge-shaped area of fibrosis containing scattered plasma cells. Elsewhere there was a mixed population of lymphocytes and plasma cells in an inconspicuous mesh of reticular cells, amongst which were scattered large, sometimes binucleated, mirror-imaged 'reticulum' cells. Reticulin fibrils were increased.

Fever began on November 22, the temperature remaining between $100^{\circ}$ and $104^{\circ} \mathrm{F}$., with rare falls to normal, until her death. No infective cause for this was found. 


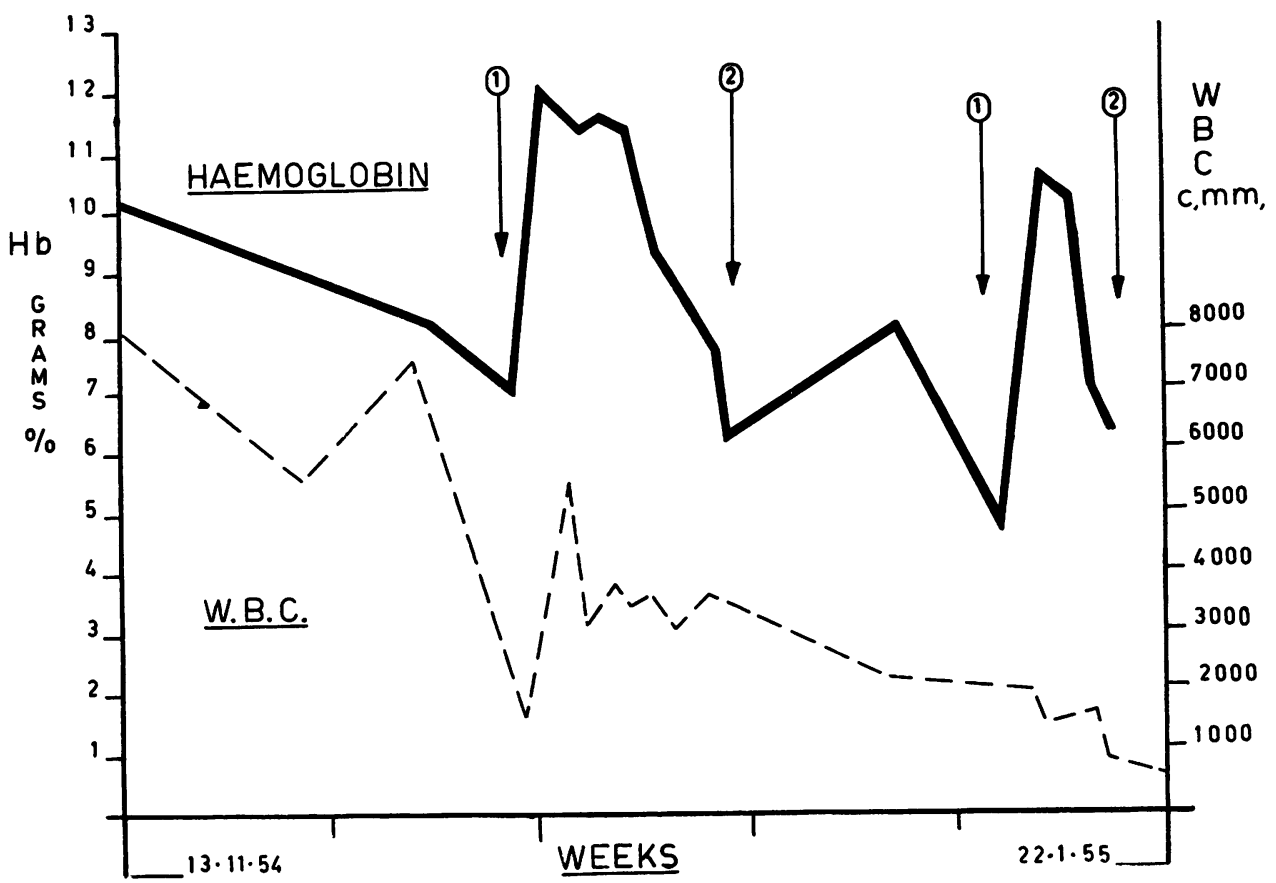

FIG. 1.-Haemoglobin and white count during final hospitalization. The numbered arrows indicate pints of transfused blood.

Blood cultures were negative and her serum did not agglutinate suspensions of Salmonella or Brucella organisms. In spite of the fever the lymph nodes and spleen began to subside on December 2, the spleen being just palpable by December 9, and the lymph nodes very small. A further aspirate of bone marrow on December 13, contained $27 \%$ monocyte-like forms, $13 \%$ lymphocytes, $41 \%$ polymorphonuclear white cells (with no excess of blast forms), $18 \%$ normoblasts and $1 \%$ plasma cells. Many of the monocytic forms had ingested red blood corpuscles, normoblasts, neutrophils and platelets (Fig. 2). A platelet count at this time was 120,000 per c.mm. A mild jaundice was noticed on December 22, and this increased steadily. By January 10, 1955 , she was extremely wasted and deeply jaundiced; the lymph nodes had again enlarged to the size of marbles and the spleen and liver were easily felt. On January 13, a month since the last platelet count, the number had fallen to 14,000 per c.mm. and did not rise above this level again. At this time purpura and bruising appeared, followed by epistaxes and bleeding from injection sites. A direct Coombs test and a test of red cell fragility, both giving negative results, were not done until a few days before death, which occurred on January 21, 1955.

Findings at Necropsy. The autopsy (A.C.H. No. 8/55) was performed 60 hours after death. The body was extremely emaciated, deeply jaundiced, with numerous petechial haemorrhages in the skin. The pleural and abdominal cavities contained clear orange effusions. The enlargement of the lymph nodes involved all groups, the cervical and axillary glands being most affected and the lower abdominal and inguinal nodes least. The cut surfaces of the glands were firm, pink to brownish purple with ill-defined yellow areas of necrosis. A few nodes were uniformly yellowish. The greatly enlarged spleen weighed $260 \mathrm{~g}$., the serosal and cut surfaces showing irregular shaped yellowish areas up to $0.5 \mathrm{~cm}$. in diameter, scattered over a dark purple background. The liver was enlarged and pale fawn, weighing $670 \mathrm{~g}$. The sternal marrow was pale. The lateral muscles of the right thigh were soft and greyish, the overlying subcutaneous tissue haemorrhagic and the skin vesiculated, these changes being considered due to haemorrhage and infection at the site of injections. Both middle ears, but particularly the right, contained greenish pus from which a Proteus organism was isolated. Other organs showed no abnormalities apart from the effects of jaundice and anaemia.

Microscopically, identical changes were found in the lymph nodes and spleen. The architecture of the organs was largely replaced by a loose-textured moderately cellular tissue in which there was much haemorrhage and coagulation necrosis. The necrotic areas, particularly in the spleen, resembled small infarcts. The cellular areas consisted of a loose apparently syncytial proliferation of reticular cells, having oval vesicular nuclei and eosinophilic cytoplasm. Amongst this were typical macrophages which varied in number from place to place and many of which contained red cells and nuclear debris (Fig. 3). Another conspicuous but less numerous element was a giant cell having a large and 


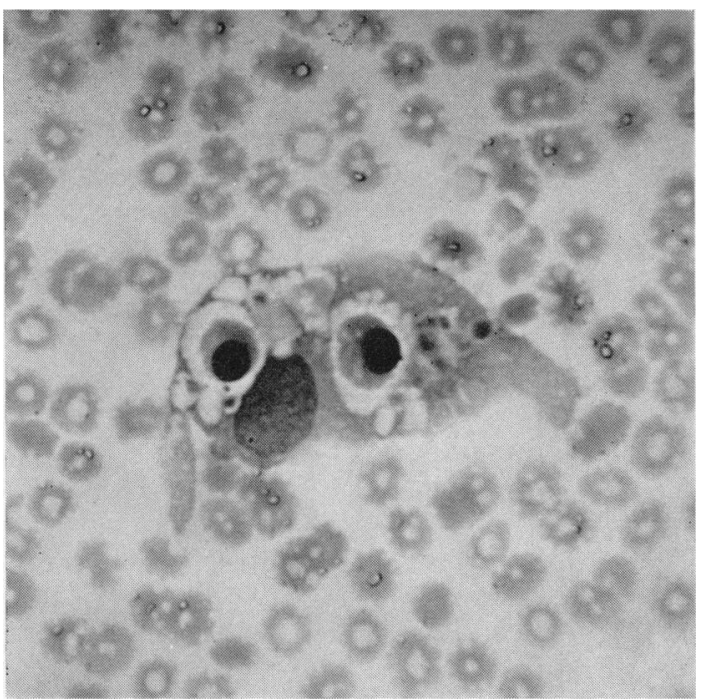

FIG. 2.-Bone marrow smear, showing a large phagocyte containing two red blood corpuscles, two normoblasts and platelets. $($ Giemsa $\times 750$.

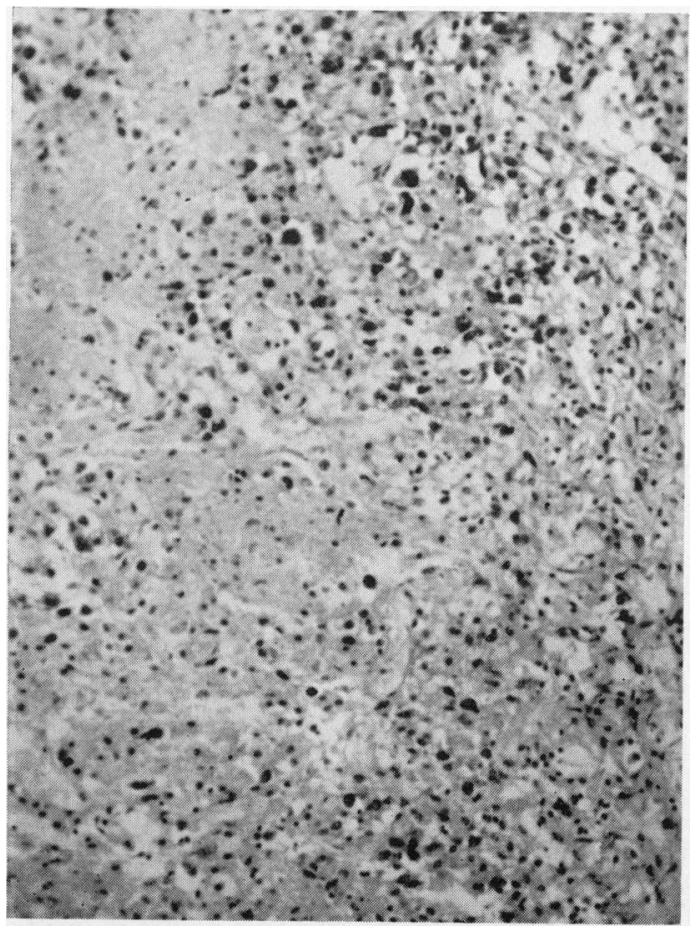

Fig. 4.-Lymph node, showing necrotic areas and densely staining giant cells.

(H. and E. $\times 150$.)

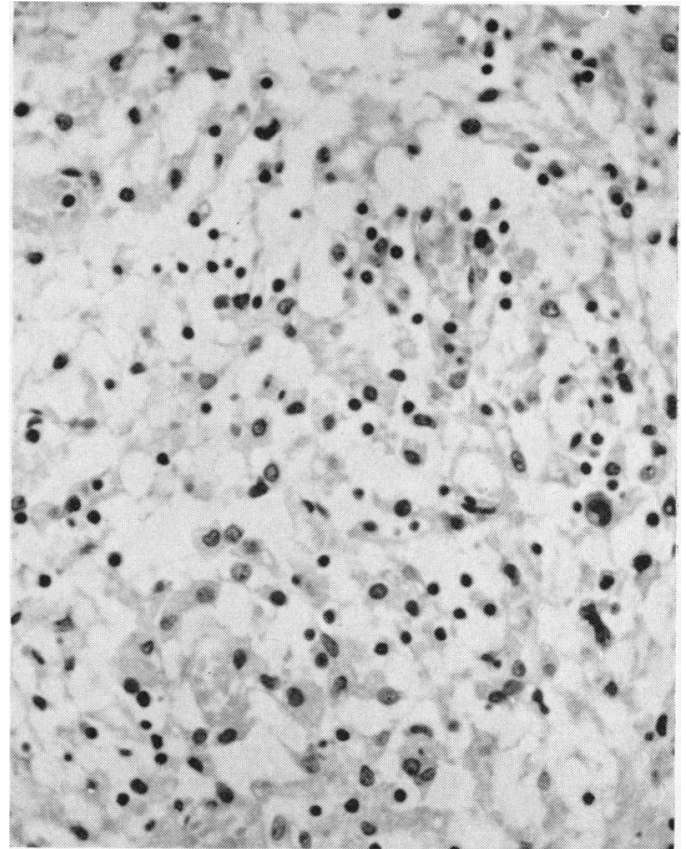

Fig. 3.-Lymph node, showing reticular arrangement of histiocytes. (H. and E. $\times 330$.)

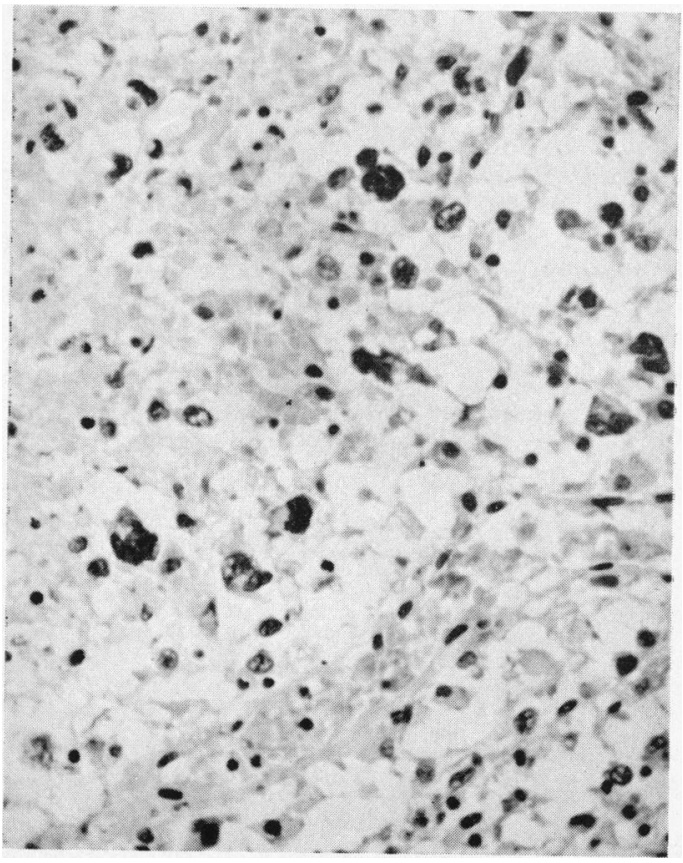

FIG. 5.-Lymph node, showing the irregular-shaped hyperchromatic nuclei of the giant cells. (H. and $\mathrm{E} . \times 330$.) 
hyperchromatic nucleus and a small amount of eosinophilic or occasionally basophilic cytoplasm (Fig. 4). The nucleus of the cell was commonly irregularly notched or folded, and if, as sometimes occurred, several nuclei were present these were closely packed together and unequal in size (Fig. 5). Transitions between these three main types of cell could be seen. Phagocytosis by the giant cells was not observed. Moderate numbers of mitotic figures were found, mostly in large cells. Mixed with this meshwork of histiocytic cells were small numbers of lymphocytes and many plasma cells and plasmablasts. A notable feature of the abnormal tissue was the large number of thin-walled blood vessels. In the lymph nodes the peripheral sinuses were usually preserved and contained many macrophages. In the spleen a few lymphoid follicles remained.

The sternal marrow showed almost complete replacement by tissue similar to that in the spleen and lymph nodes, except that giant cells were rare.

The liver had undergone a moderate degree of fatty change and congestion, and in addition contained occasional small haemorrhagic and necrotic foci of histiocytes and giant cells. Similar groups of tumour cells were found in the suprarenal cortex. Phagocytosis of red cells by Kupffer cells was present throughout the liver.

Other organs contained no significant changes. Perl's stain for iron revealed much haemosiderin in the spleen and Kupffer cells, but very little in the lymph nodes. Reticulin fibrils were increased in the lymph nodes.

\section{Discussion}

The clinical and pathological findings in this case conform in every way to the previous descriptions of the disease, except for the age of the patient. It is not proposed here to discuss the relation of this condition to other reticuloses; the pathological findings in the reticulo-endothelial organs are, however, quite singular. Nevertheless, the histological changes in the cervical lymph gland of the present case were considered by several pathologists to be typical of classical Hodgkin's disease, appearances which were to be completely replaced by the time of death two months later.
The relapsing nature of the illness also calls for attention, indicating that the course of the disease cannot be fully explained by neoplastic proliferation. The sudden enlargement of the spleen and lymph nodes may have been partly due to vascular engorgement rather than cellular proliferation alone. The equally sudden return of these organs to relatively normal size is hard to explain on other grounds.

The preliminary diagnosis of infectious mononucleosis could not be maintained, particularly as the Paul-Bunnell tests were performed with nonabsorbed sera.

The rapid fall of the haemoglobin level following transfusions (see Fig. 1) suggested that the anaemia was due not solely to replacement of the bone marrow and this was confirmed pathologically by the discovery of widespread erythrophagocytosis and haemorrhage.

\section{Summary}

A girl of $4 \frac{1}{2}$ years presented with enlargement of the cervical lymph nodes and spleen, followed by anaemia, leucopenia, fever and enlargement of the liver. Jaundice, thrombocytopenia and a bleeding tendency appeared terminally.

A lymph node removed during life showed appearances typical of Hodgkin's disease but at death the reticulo-endothelial system was replaced by a proliferation of phagocytic histiocytes and giant cells.

I am grateful to Dr. Malcolm Cockburn for the use of the case record and to the Board of the Adelaide Children's Hospital for permission to publish the material. Thanks are due to Mr. Ray Boyd for the preparation of the illustrations.

\section{REFERENCES}

Marshall, A H E (1956a). An Outline of the Cytology and Pathology of the Reticular Tissue. Oliver and Boyd, London. (1956b). Histiocytic medullary reticulosis. J. Path. Bact., 71,61 .

Scott, R. B. and Robb-Smith, A. H. T. (1939). Histiocytic medullary reticulosis. Lancet, 2, 194 